\title{
FECUNDIDADE E AMBIENTE: TEMAS E CONTROVÉRSIAS
}

\author{
César Marques* \\ Angelita Alves Carvalho**
}

Resumo: A dificuldade em encontrar nexos causais entre a fecundidade e a mudança ambiental sempre esteve presente nas pesquisas demográficas. As relações entre tais variáveis são de difícil apreensão e são raras as referências bibliográficas no tema. Neste artigo buscamos sintetizar essa discussão em dois elementos principais: tratamos das influências ambientais sobre o comportamento reprodutivo e da influência da fecundidade sobre o ambiente, enfatizando a discussão pró-natalista e controlista. Nesse sentido apontamos que as principais justificativas ambientais para tais iniciativas acabam obscurecendo as demandas por direitos reprodutivos.

Palavras-chave: Fecundidade. Políticas de população. Mudanças ambientais.

Fertility and Environment: issues and controversies

Abstract: Causal links between fertility and environmental change are hardly established in demographic research. The relationship among these variables are difficult to grasp and there are few explicit references on the subject. In this article, we seek to summarize this discussion in two main elements: dealing with environmental influences on the reproductive behavior and with the influence of fertility on the environment, emphasizing the pro-natalist and population control discussion. We point out that the main environmental reasons for such initiatives end up obscuring the demands for reproductive rights.

Keywords: Fertility. Population policies. Environmental change.

Fecundidad y Medio Ambiente: temas y controversias

Resumen: La dificultad de encontrar nexos causales entre la fecundidad y el cambio ambiental siempre ha estado presente en las investigaciones demográficas. Las

* Escola Nacional de Ciências Estatísticas - ENCE/IBGE, Rio de Janeiro-RJ, Brasil (cesar.m.silva@ibge.gov.br).

** Escola Nacional de Ciências Estatísticas - ENCE/IBGE, Rio de Janeiro-RJ, Brasil (angelita.carvalho@ibge.gov.br).

Recebido em: 30/01/2015 - Aceito em: 02/07/2015. 
$|14|$

Fecundidade e ambiente..

relaciones entre dichas variables son de difícil comprensión y hay raras referencias bibliográficas sobre el tema. En este artículo tratamos de sintetizar esta discusión en dos elementos principales: tratamos de las influencias ambientales sobre el comportamiento reproductivo y la influencia de la fecundidad en el medio ambiente, enfatizando la discusión pro-natalista y controlista. En este sentido señalamos que las principales justificaciones ambientales para tales iniciativas terminan oscureciendo las demandas por los derechos reproductivos.

Palabras clave: Fecundidad. Políticas de población. Cambios ambientales.

\section{Introdução}

A área de população e ambiente já possui hoje mais de duas décadas de pesquisas que tratam da interface entre dinâmica populacional e mudança ambiental (HOGAN, 2007). Possui perspectivas teóricas e metodológicas próprias e críticas e está relativamente bem consolidada, com quantidade de estudos de qualidade relevante e em expansão (LUTZ et al., 2002; HUMMEL et al., 2012).

Grande parte desses avanços estão documentados na principal revista do campo, Population and Environment, criada em 1978 e com 36 volumes publicados até 2014 . No seu site', uma busca simples usando a palavra "fertility" gerou um resultado, de certo modo, surpreende. Foram 479 artigos, mas somente 65 identificados no grupo das ciências ambientais. Apesar da revista possuir abertura para o tema, uma pequena parte dos artigos debate as interações específicas entre população e ambiente a partir da ótica da fecundidade.

Nesse contexto, o objetivo desse artigo é apresentar uma revisão das principais discussões que tratam da relação entre fecundidade e ambiente, explorando as dificuldades em relacionar as influências do ambiente sobre a fecundidade e analisando em que medida os diferentes cenários de fecundidade no Brasil e no contexto europeu (com políticas pró-natalistas) influenciariam

\footnotetext{
${ }^{1}$ Disponível em: http://link.springer.com/journal/11111. Acesso e pesquisas realizados em: 19 jan. 2015.
} 
as questões ambientais. Para isso utilizaremos as projeções populacionais das Nações Unidas (UN, 2013).

O intuito de tal exercício é relativizar os argumentos contrários às políticas pró-natalistas, que enfatizam excessivamente o tamanho populacional como um dos elementos que minimizariam a mudança climática e os problemas ambientais, já que as mudanças na fecundidade em contextos de baixíssima fecundidade não seriam determinantes nas suas respectivas pegadas ecológicas.

\section{As influências do ambiente na fecundidade}

\section{Dinâmicas rurais}

O comportamento da fecundidade é influenciado por dimensões sociais, políticas, econômicas e ambientais, dentre outras. A partir de diferentes mecanismos o ambiente é mais um dos contextos que potencialmente influenciam a fecundidade. No entanto, tal relação tem sido mais abordada em pesquisas na escala local focadas na dinâmica rural, onde é possível melhor observar tais relações.

Uma das correntes do tema argumenta que há um processo recíproco, com efeito negativo do crescimento populacional sobre a qualidade ambiental, e por outro lado, com a degradação ambiental contribuindo para o crescimento populacional (BIDDLECOM et al., 2005 AGGARWAL et al., 2001; FILMER e PRITCHETT, 2002). Nesse modelo a pobreza leva à alta fecundidade em função de uma série de fatores, como a demanda por mão de obra agrícola, os incrementos de segurança para os pais na velhice, a alta mortalidade infantil e o baixo empoderamento das mulheres. A fecundidade elevada leva ao crescimento da população, e, consequentemente, da demanda por alimentos e recursos. Nesse contexto, o declínio da disponibilidade per capita dos recursos reforça a pobreza pela perda de fertilidade do solo e dos rendimentos decrescentes. A pobreza, por sua vez, contribui à degradação da terra, pela exploração de curto prazo e uso inadequado de fertilizantes 
$|16|$

Fecundidade e ambiente..

e tecnologias (LUTZ \& SCHERBOV, 1999; O'NEILL et al., 2001; DE SHERBININ et al., 2007). Forma-se assim um "círculo vicioso" entre altos níveis de fecundidade e piora das condições ambientais (AGGARWAL et al 2001; FILMER e PRITCHETT, 2002).

Este modelo é representado na Figura 1, que descreve os mecanismos de interação entre o contexto ambiental, familiar e da reprodução (BRAUNER-OTTO, 2014).

A aplicação do modelo, feita em áreas rurais do Nepal, encontrou uma relação positiva entre densidade de plantas, riqueza de espécies, diversidade vegetal e o momento do uso de contraceptivos. As mulheres inseridas em contextos de piores condições ambientais foram menos propensas a encerrar a vida reprodutiva, ou fazê-lo posteriormente, e, portanto, mais propensas a ter famílias maiores. Nesse caso, as funções da criança associadas à sobrevivência do agregado familiar também foi favorável a um maior número de filhos, o que influenciou mais na relação ambiente-fecundidade do que os mecanismos biológicos. Além disso, se evidenciou que o comportamento reprodutivo dependeu de outros recursos contextuais, como a disponibilidade de serviços de saúde e o acesso a contraceptivos (BRAUNER-OTTO, 2014). 
FIGURA 1 - Representação do efeito da má qualidade ambiental na família e fecundidade

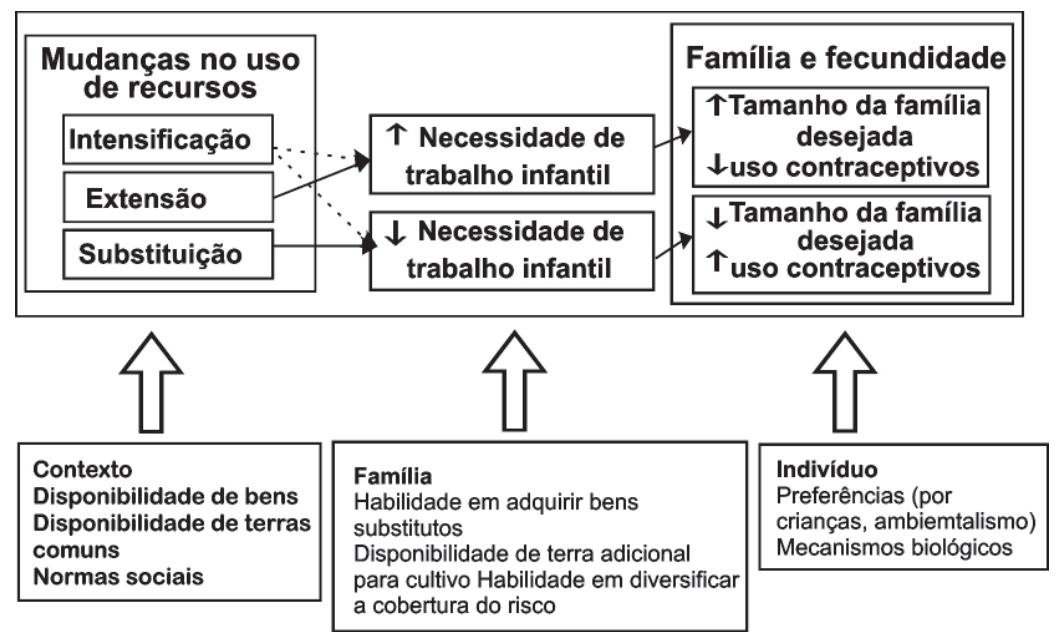

Fonte: Adaptado de Brauner-Otto, 2014. As representações não são mutuamente exclusivas. As linhas pontilhadas representam possibilidades teóricas conflitivas.

Na Amazônia brasileira, Perez (2001) explorou a relação entre ambiente e desejo por filhos entre pecuaristas em regiões de fronteira (onde não há extrema pobreza). Nestes casos a obtenção do capital para compra dos insumos à produção tende a ocorrer em idades mais avançadas, pois a acumulação de capital suficiente ocorre após o agricultor ter filhos e estes fornecerem remessas financeiras. De acordo com essa hipótese os pais demandam filhos pela sua capacidade de trabalho na produção agrícola quando crianças e pelas remessas quando adultos (PEREZ, 2001). Ainda para a Amazônia, VanWey et al. (2007) demonstram que as mudanças no número de crianças e mulheres, mais do que o número de homens adultos, exercem influências mais significativas sobre as mudanças no uso e cobertura da terra. Resultados similares foram encontrados nas regiões de fronteira da Guatemala, com 
| 18 |

Fecundidade e ambiente..

a especificidade de que o tipo de trabalho no campo é importante para os padrões de fecundidade (SUTHERLAND et al, 2004).

\section{Desastres, mortalidade e fecundidade}

Uma das conexões entre ambiente e fecundidade está na relação mortalidade-fecundidade. Essa ocorre onde há baixa expectativa de sobrevivência das crianças, e com isso, necessidade de reposição do óbito infantil. Nesses casos, haveria dois mecanismos: um de "segurança", em contextos de mortalidade infantil e probabilidade alta de perda de criança, com um efeito ex ante; e um mecanismo de reposição, com um efeito ex post, de gestação de uma nova criança para repor a morte de outro filho (MONTGOMERY e COHEN, 1998).

Nesse sentido, desastres, guerras, crises de fome e ambientais que influenciam a mortalidade de modo pontual podem levar a alterações no comportamento reprodutivo em função do impacto na mortalidade infantil (HILL, 2004; CALDWELL, 2004; AGADJANIAN e PRATA, 2002; FINLAY, 2009).

Em três estudos de caso sobre terremotos (Turquia - 1999, Índia - 2001, Paquistão - 2005), Finlay (2009) evidencia que houve uma resposta positiva da fecundidade à exposição aos desastres e à mortalidade infantil por eles geradas. Indo além, com a importância do contexto em que o choque ocorre e das normas culturais relativas às crianças, o aumento da fecundidade foi uma resposta a perdas não só de crianças, mas também de renda, dado o papel das crianças em relação ao trabalho e à renda.

Em pesquisa na Indonésia sobre os impactos do Tsunami de 2004, Nobles, Frankenberg e Thomas (2015), também concluem que houve resposta positiva da fecundidade em função da exposição à mortalidade. De modo geral a fecundidade aumentou após o Tsunami em função da maior propensão de mães que perderam filhos terem novas gestações e das mulheres que não possuíam filhos iniciar a reprodução antecipadamente. 


\section{Os debates da saúde e reprodução: ambiente e Infertilidade}

Além das profundas mudanças sociais do século XXI que resultaram na queda acentuada das taxas de natalidade (LUTZ et al., 2003), também se argumenta que esta tendência pode estar relacionada à perda de fertilidade (SKAKKEBAEK et al., 2001).

Essa tendência pode afetar especialmente os homens, já que a produção dos espermatozoides é contínua e mais sujeita aos fatores ambientais, (UFJF, 2011). Em São Paulo, por exemplo, houve uma diminuição da razão de sexo ${ }^{2}$ em função da poluição atmosférica, com aumento relativo dos nascimentos de mulheres em áreas mais poluídas (LICHTENFELS et al., 2007).

Ainda que não exista consenso sobre os determinantes na infertilidade masculina, diversos fatores têm contribuído para esse quadro, como os comportamentais, biológicos, socioeconômicos e físicos (PARADISI, et al., 2009; UFJF, 2011).

A baixa qualidade do esperma e o aumento de anomalias geniturinárias teria uma etiologia comum com o ambiente, onde podem atuar diversos agentes deletérios para o sistema reprodutor, seja via exposição ocupacional, doméstica, recreacional ou ambiental (PARADA et al., 2004).

Burdorf et al. (2006) mostrou os efeitos da exposição ocupacional a pesticidas na reprodução, por exemplo, bem como os efeitos de substâncias químicas ambientais na fertilidade da população em geral. Para Koifman e Hatagima (2003) um grande número de agrotóxicos tem o potencial de desregular o equilíbrio endócrino de seres humanos e animais, levando a cânceres, a modificação na razão de sexos ao nascimento, infertilidade, másformações congênitas no trato genital masculino e a modificações na qualidade do sêmen. Já Levine et al. (1988) e Tas et al. (1996) mostram que há relação entre as temperaturas elevadas de determinados locais de trabalho e a diminuição da densidade do esperma. No Brasil, Andrietta (2010) indicou que a poluição veicular

2 A razão de sexo expressa a quantidade de homens para cada 100 mulheres. 
afetou negativamente a saúde reprodutiva de controladores de tráfico em São Paulo.

Contrariamente, na Europa se argumenta que a melhoria do ambiente devido a regulamentações mais rígidas sobre o uso de produtos tóxicos e as mudanças no estilo de vida, com a diminuição do tabagismo e o declínio das Doenças Sexualmente Transmissíveis, podem ter mudado a fertilidade da população (SCHEIKE et al., 2008). No entanto, a crescente epidemia de obesidade e o adiamento da maternidade para as idades em que homens e mulheres se tornam menos férteis devem ter um impacto negativo sobre os níveis de fecundidade (HOMAN et al., 2007). E, mais do que problemas genéticos, ainda se aponta que os principais responsáveis pelo aumento da infertilidade ainda são os fatores ambientais - como a exposição à poluição - e o estilo de vida não saudável (HAUSER et al., 2005).

\section{Fecundidade, ambiente e consumo: existe um ponto ótimo?}

Não há consenso sobre qual tamanho populacional e quais deveriam ser as taxas de fecundidade ideais para a sustentabilidade no planeta. Contudo, mesmo se isso fosse possível, seria também necessário conhecer os níveis e padrões de consumo que o mundo suporta.

Desde a década de 1960, quando a população global atingiu suas maiores taxas de crescimento e a questão ambiental ganhou força, várias obras relataram a preocupação com a questão populacional. Ehrlich (1968) argumentou que o rápido crescimento populacional levaria o mundo a uma série de crises com escassez generalizada de recursos. Meadows et al. (1972) destacaram que mantidos os ritmos do crescimento econômico e populacional haveria um colapso global dos sistemas econômicos e sociais em meados do século XXI. Cohen (1995) colocou a pergunta "Quantas pessoas o mundo pode suportar?" como uma questão útil mas totalmente incompleta, já que não incorpora as mudanças econômicas e tecnológicas. Nessa linha Boserup (1981) 
argumenta que o crescimento da população induz a inovações tecnológicas que podem evitar ou atenuar a degradação. Nesse sentido, se uma menor população ajudará a resolver os problemas ambientais (ROYAL SOCIETY, 2012), sua diminuição não garante esse resultado. Esse debate é extremamente amplo, e não cabe aqui nos aprofundarmos nele, mas sim perceber que nele residem as maiores implicações para a relação fecundidade-ambiente.

Considerando questões ambientais e econômicas, Striessnig e Lutz (2014) apontaram um possível ótimo de fecundidade calculado a partir de preocupações com o envelhecimento populacional e com a questão ambiental (especificamente a climática). Em relação à primeira, a queda da fecundidade modifica a estrutura etária, que passa a ter maior participação dos idosos na população, pressionando os sistemas de seguridade social. Para a segunda dinâmica admite-se que não haveria relação direta entre fecundidade e emissões de carbono, já que as emissões são intermediadas por outras dinâmicas, como a urbanização. Com uma proposta de pesos (no máximo 20\% para a dimensão ambiental e mínimo de $80 \%$ para a dimensão social do envelhecimento), chegam a uma TFT ideal entre 1,41 e 1,51 para a Europa e China.

Há, certamente, outros critérios para a construção de um possível nível ideal de fecundidade. Da perspectiva individual, o "ótimo" pode ser relativo ao desejo supostamente "natural" de ter dois filhos e assim substituir o casal (LUTZ e SCHERBOV, 2008). No entanto, a obtenção da fecundidade desejada pela família produz um nível agregado nem sempre desejado pelos países. Para esses a população é um fator de segurança relevante onde, historicamente, maiores taxas de fecundidade, que permitam algum crescimento da população, são preferíveis. Como tais critérios (no nível dos países e dos indivíduos) continuam a desempenhar um papel importante, não há tendências de que seja possível operacionalizar internacionalmente critérios para uma fecundidade ideal (STRIESSNIG e LUTZ, 2014).

O consumo é um ponto central nessa discussão, já que a sustentabilidade não depende exclusivamente do tamanho 
populacional. Como Curran e De Sherbinin (2004) analisam, o tema pode e deve ser incorporado nos estudos de população e ambiente, já que as cadeias de produção e consumo afetam diretamente as questões ambientais, especialmente dos países desenvolvidos, onde o estilo de vida e o consumo elevado tem acarretado na degradação ambiental global (HUNTER, 2000).

Esse debate se divide em dois pontos de vista, uma vez que as pressões ambientais podem se tornar mais intensas tanto nos países de maior crescimento populacional (onde há menor renda e altas taxas de pobreza), com degradação decorrente da baixa disponibilidade de recursos per capita; como também entre os países mais desenvolvidos, com baixo crescimento populacional, mas níveis elevados de rendimento, de consumo energético e da produção de resíduos. Estas diferentes ênfases apontam, naturalmente, para diferentes soluções: por um lado defende-se um lento crescimento populacional em países menos desenvolvidos a partir da implantação de políticas de planejamento familiar eficaz e, por outro, se esperam ações que levem a mudanças nos padrões de produção e consumo destrutivo das nações mais desenvolvidas (HUNTER, 2001).

Essas soluções parecem pouco prováveis nos cenários atuais, principalmente em contextos de baixa e baixíssima fecundidade, onde, ao contrário, atenção tem sido dada às políticas pró-natalistas e pouco se tem avançado em questões relativas aos níveis e padrões de consumo.

\section{Políticas pró-natalistas: fundamentos e limites}

A crescente preocupação sobre as implicações do envelhecimento da população estimulam o interesse para incentivos que visem o aumento da fecundidade. Considerando a tendência de queda da fecundidade durante os próximos anos, Lutz e Skirbekk (2005) discutem a possibilidade de uma "armadilha da baixa fecundidade" (que chega a níveis de difícil reversão), chamando a atenção para a necessidade de implementação 
imediata de políticas que impulsionem seus níveis à curto prazo. Tais políticas podem minimizar os custos de oportunidade das mulheres, bem como o conflito entre a família e as demandas de emprego, e assim influenciar o processo cognitivo e emocional por trás das intenções de fecundidade (PHILIPOV et al., 2009).

As políticas pró-natalistas são pensadas porque normalmente a baixa fecundidade está associada a uma discrepância entre o número de filhos desejados e tidos, onde há uma possível incapacidade de alcançar a fecundidade desejada. Portanto, assim como em contextos de fecundidade maior do que a desejada, há restrições à implementação dos direitos reprodutivos (BONGAARTS, 2001; PHILIPOV et al, 2009).

Em sua maioria, tais políticas são focadas em auxílios no custo de criação dos filhos, incentivado a participação na força de trabalho e no aumento da igualdade de gênero (GAUTHIER e PHILIPOV, 2008), com transferências de renda ou subsídios fiscais para famílias com filhos, compensando o custo das crianças (BILLINGSLEY e FERRARINI, 2014). Para incentivar a participação na força de trabalho, há a licença remunerada para mães e pais e provisão pública de centros de atendimento às crianças. Já as políticas para o aumento da igualdade de gênero incluem rendimentos relacionados a benefícios de licença (especificamente de paternidade), a prestação de assistência à infância e a ausência de subsídios fiscais de casamento. Estas políticas cobrem aspectos fundamentais para a tomada de decisões de fecundidade, embora outras motivações, como o aumento da equidade, possam existir. No estudo sobre políticas em prol do aumento da fecundidade em 21 países europeus, os autores mostraram que essas são variadas, mas que todas resultaram em aumento das intenções para o primeiro nascimento, em menor medida para o segundo e, em geral, não se relacionaram às intenções de paridade de terceira e demais ordens (GAUTHIER e PHILIPOV, 2008).

Com proposito semelhante, o trabalho de Luci-Greulich e Thévenon (2013) testou o impacto das políticas de família em 18 países da OCDE entre 1982 e 2007, concluindo que seus diversos instrumentos e pacotes tiveram, no geral, influências positivas 
para o aumento da fecundidade. Contudo, a eficácia no aumento da fecundidade foi maior com a combinação de um conjunto de medidas, de curto prazo, antes e após o nascimento da criança, e de longo prazo, durante seu desenvolvimento.

Segundo Martine (2014), como normalmente os grandes problemas ambientais são diretamente relacionados ao tamanho populacional, à primeira vista muitas destas políticas que visam o aumento da fecundidade podem contribuir para a degradação ambiental. Entretanto, é essencial considerar que são os aumentos de riqueza, e não de população, que explicam os incrementos de produção e consumo global, esses sim, insustentáveis ambientalmente. Uma pessoa não é necessariamente uma unidade de consumo e apenas um terço da população mundial contribui para a emissão de poluentes. Além disso, a redução de fecundidade é quase sempre ligada a melhorias nas condições de vida com aumento do consumo. Desse modo, a queda da fecundidade é uma variável importante, mas a discussão sobre o consumo é essencial (MARTINE, 2014).

\section{Cenários de fecundidade e crescimento populacional}

Em 2010 a fecundidade do mundo como um todo foi estimada em 2,53 filhos por mulher (UN, 2013). De 201 países, 74 tiveram taxas abaixo do nível de reposição $(2,1)$ e 33 abaixo de 1,5 . A Europa teve fecundidade de 1,54, a América do Norte de 2,02 e as regiões mais desenvolvidas do mundo, de 1,67 (UN, 2013). Curiosamente são nos países com baixa fecundidade que surgem algumas iniciativas que colocam a população como uma "vilã" do ambiente, sugerindo que a fecundidade declina.

Alguns exemplos são o grupo GINK - Green Inclination, No Kids, em alusão aos casais DINK (Doule Income, No Kids) e os VHEMT (The Voluntary Human Extinction Movement). ${ }^{3}$. Os GINK defendem que a opção por não possuir filhos impede que seja criado

\footnotetext{
${ }^{3}$ Disponível em: http://www.vhemt.org/. Acesso em: 19 jan. 2015.
} 
um "legado" de emissão de carbono, dos filhos e dos respectivos descendentes. A conta da economia nas emissões é grande, já que envolve todo o ciclo de vida de várias gerações (HYMAS, 2011). Dunlop (2013) critica o grupo, mostrando que esses não focam questões centrais e privilegiam ações individuais em detrimento às ações de grandes instituições (como companhias petrolíferas e militares), ameaçando direitos reprodutivos e a justiça climática. Já os VHEMT trazem como slogan o lema "que possamos ter uma vida longa e morrer". Sua premissa é de que as pessoas devem parar de se reproduzir, ou seja, que nenhum ser humano seja adicionado à Terra, levando à extinção da raça humana para que a biodiversidade como um todo seja mantida.

De outro lado também ganha força no mundo desenvolvido a defesa de políticas pró-natalistas que consigam elevar as taxas de fecundidade em contextos de rápido envelhecimento populacional. Mas qual o impacto dessas mudanças em termos globais? As projeções demográficas mais recentes das Nações Unidas ${ }^{4}$ podem dar alguns indícios para responder à questão.

Utilizando tais projeções, qual será o impacto do aumento ou decréscimo da fecundidade no crescimento populacional durante o século XXI na Europa? E no Brasil? A Tabela 1 traz uma síntese desses dados para a Europa, dois países desse continente (França e Itália) e para o Brasil.

\footnotetext{
4 É importante observar as inovações metodológicas de tais projeções, que utilizam estimativas probabilísticas baseadas em modelos bayesianos hierárquicos a partir das evidências empíricas dos componentes demográficas de cada um dos países e do conjunto de países. Como modelo probabilístico, podem-se calcular dezenas de milhares de diferentes projeções, e para essas, calcular intervalos de predição (de $80 \%$ e $95 \%$ ), que quantificam incertezas e o nível de confiança (RAFTERY et al., 2012; FOSDICK e RAFTERY, 2014).
} 
TABELA 1 - População, TFT e Razão de Dependência Total para regiões selecionadas, $\mathbf{2 0 1 0 - 2 1 0 0}$

\begin{tabular}{|c|c|c|c|c|c|c|c|}
\hline \multirow[t]{2}{*}{$\begin{array}{c}\text { País/ } \\
\text { Região }\end{array}$} & \multirow{2}{*}{$\begin{array}{c}\text { Variável } \\
\text { da } \\
\text { projeção¥ }\end{array}$} & \multicolumn{2}{|c|}{$\begin{array}{c}\text { População } \\
\text { (Em milhões) }\end{array}$} & \multicolumn{2}{|c|}{$\begin{array}{c}\text { Taxa de } \\
\text { Fecundidade } \\
\text { Total }(\mathrm{TFT})^{\mathrm{p}}\end{array}$} & \multicolumn{2}{|c|}{$\begin{array}{c}\text { Razão de } \\
\text { Dependência } \\
\text { Total (RDT) }\end{array}$} \\
\hline & & 2010 & 2100 & 2010 & 2100 & 2010 & 2100 \\
\hline \multirow{3}{*}{ Europa } & Mediana & \multirow{3}{*}{740,3} & 638,8 & \multirow{3}{*}{1,54} & * & \multirow{3}{*}{46,5} & 79,6 \\
\hline & $95 \%$ & & 737,2 & & * & & 85,6 \\
\hline & $-95 \%$ & & 548,9 & & * & & 74,8 \\
\hline \multirow{3}{*}{ Itália } & Mediana & \multirow{3}{*}{60,5} & 54,6 & \multirow{3}{*}{1,29} & 1,88 & \multirow{3}{*}{52,3} & 89,7 \\
\hline & $95 \%$ & & 69,8 & & 2,32 & & 110,9 \\
\hline & $-95 \%$ & & 38,6 & & 1,36 & & 76,5 \\
\hline \multirow{3}{*}{ França } & Mediana & \multirow{3}{*}{63,2} & 69,8 & \multirow{3}{*}{1,79} & 1,99 & \multirow{3}{*}{54,2} & 84,5 \\
\hline & $95 \%$ & & 103 & & 2,46 & & 99,8 \\
\hline & $-95 \%$ & & 62,1 & & 1,58 & & 73,3 \\
\hline \multirow{3}{*}{ Brasil } & Mediana & \multirow{3}{*}{195,2} & 194,5 & \multirow{3}{*}{1,82} & 1,83 & \multirow{3}{*}{47,9} & 86,9 \\
\hline & $95 \%$ & & 308,5 & & 2,28 & & 166,1 \\
\hline & $-95 \%$ & & 98,4 & & 1,46 & & 65,5 \\
\hline
\end{tabular}

Fonte: UN(2013).

$¥$ As projeções recentes das Nações Unidas são feitas a partir de milhares de simulações possíveis, das quais três valores são utilizados como cenários: um limite superior dos valores da fecundidade (que corresponde ao valor que engloba o limite de $+95 \%$ em relação a mediana), um valor limite inferior (-95\% em relação a mediana) e a mediana de todas as projeções (UN, 2013).

* Os valores projetados da TFT não estão disponíveis.

pA TFT corresponde ao número médio de filhos por mulher ao final da idade reprodutiva em um ano-calendário.

A RDT é razão entre a soma da população considerada dependente (entre 0 e 14 anos de idade e os maiores de 65 anos) pela população considerada ativa (15 a 64 anos). 
A Itália foi um dos primeiros países a possuir baixíssima fecundidade. De acordo com as projeções, a fecundidade de 2010, de 1,3, flutuaria entre 1,36 e 2,32 até 2100, com mediana de 1,88 . Sua população, estimada em 60,5 milhões de habitantes em 2010, chegaria a 61,3 milhões em 2020 e então declinaria a 54,6 milhões em 2100 no cenário da mediana. Mesmo com elevação da fecundidade esse cenário resultaria em decréscimo populacional já nos próximos anos (UN, 2013).

Já na França, que possui uma TFT mais elevada, no cenário da mediana a fecundidade em 2100 seria superior a de 2010, chegando a 1,98, com mínimo de 1,58 e máximo de 2,46 . Sua população estimada em 2010 é de 63,2 milhões. O resultado das projeções é que, em 2100, a população mínima seria de 62 milhões, o valor mediano de 79 milhões e o valor máximo de 103 milhões.

Na Europa como um todo a população já está muito perto do seu volume máximo, com um potencial declínio para os próximos anos. O potencial pico da população europeia é de 743 milhões em 2015, que segundo os dados da mediana da projeção chegará a aproximadamente 640 milhões em 2100, com limites mínimos e máximo de 549 e 737 milhões, respectivamente (que agrega 95\% de todos os resultados). Nesse sentido, as chances de que a população europeia do final do século seja superior a sua população atual é menor que 5\%, conforme a Figura 2 ilustra. 
FIGURA 2 - Volume da população da Europa segundo intervalos das projeções, 2010-2100

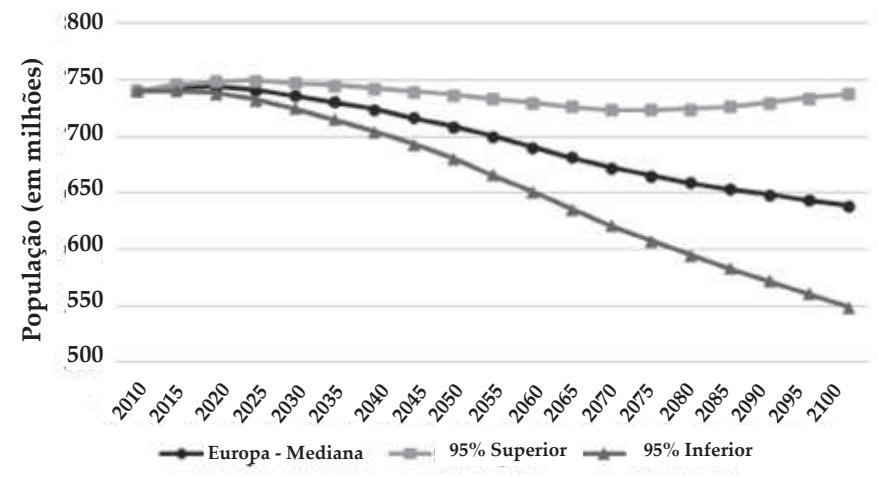

Fonte: UN (2013).

Tais projeções resultam em razões de dependência que, em todos os casos, serão superiores às atuais. Basicamente, seguem a seguinte tendência: em 2100, com fecundidade próxima a mediana, em todos os locais a RDT ficará em torno de 80, se a fecundidade atingir os valores mais altos essa razão aumentará, e caso a fecundidade caia aos menores níveis esperados a razão também diminuirá. Isso porque o aumento da fecundidade eleva o número de crianças e, consequentemente, gera aumento da razão de dependência. Por outro lado, se a fecundidade permanecer em níveis baixos, o número de crianças será menor e assim também as RDT. No entanto, a tendência é inversa se observarmos somente as razões de dependência dos idosos, que não contabilizam os menores de 14 anos.

No Brasil, com TFT mediana em 2100 de 1,83, mínima de 1,46 e máxima de 2,28 , as oscilações, tanto do total populacional como das RDT, serão maiores. Caso a fecundidade atinja seus menores valores a população será de menos de 100 milhões em 2100, e caso ela volte a subir, será superior a 300 milhões. As RDT podem chegar a 166 (ou seja, 166 dependentes para 100 pessoas na 
População em Idade Ativa), caso a fecundidade atinja os valores superiores da projeção. Para o cenário da mediana a população brasileira já se encontra acima da população projetada para 2100, sendo que o pico deverá ocorrer em 2050, quando haverá 231 milhões de habitantes.

\section{Considerações finais}

As conexões entre fecundidade e ambiente são complexas e de difícil apreensão. Há uma série de relações potenciais, como: mudanças ambientais $\rightarrow$ mortalidade $\rightarrow$ fecundidade, saúde $\rightarrow$ fertilidade $\rightarrow$ fecundidade e fecundidade $\rightarrow$ ambiente. Embora as influências do ambiente sobre a fecundidade existam, essas são mais explícitas em estudos demográficos sobre contextos rurais e nas pesquisas médicas sobre (in)fertilidade, enquanto as maiores polêmicas recaem sobre o efeito da fecundidade no ambiente em função do crescimento populacional. A conexão mais clara dessa relação está no impacto da fecundidade sobre o tamanho populacional, que tem um papel na pressão sobre os recursos. Nesse sentido não é exatamente a fecundidade que se configura como um problema, mas sim o total da população, e sobretudo, seu padrão de consumo.

Ambos os lados são perigosos nessa discussão. Os que advogam pelo controle populacional associado à saúde ambiental encorajam políticas radicais de controle populacional. Os que defendem que o crescimento populacional não importa para o equilíbrio ecológico podem perder de vista que ainda há um contingente considerável de populações com fecundidade alta devido à falta de acesso a métodos contraceptivos (CAVENAGHI, 2013). Em ambos os casos se negligenciam os direitos reprodutivos.

O primeiro grupo corre o risco de cair no individualismo absoluto sem que considere as raízes da crise ambiental. O segundo pode pensar as raízes relacionadas ao tipo de desenvolvimento econômico sem considerar as demandas não atendidas e relacionadas à pobreza, igualdade de gênero e justiça social. 
$|30|$

Fecundidade e ambiente..

No caso europeu, considerar que as políticas pró-natalistas serão responsáveis por um aumento da população que agravará as condições ambientais ou as mudanças climáticas, ou o contrário, que as políticas controlistas serão a solução do problema, significa argumentar que uma variação de até 100 milhões de pessoas resolverá a questão. Isso considerando que mesmo o valor máximo da população em 2100 será menor que o atual. Nesse contexto, o maior risco de políticas direcionadas a uma perspectiva dos direitos sexuais e reprodutivos é o de que a população decresça mais lentamente, o que, ceteris paribus, pouco interferirá na degradação ou recuperação ambiental.

Já no contexto brasileiro as diferenças entre os máximos e mínimos populacionais decorrentes das mudanças na fecundidade são relativamente maiores, com um nível ainda bastante amplo de incertezas. As projeções para 2100 variam entre 98,4 e 308,5 milhões, mas os valores da mediana já são inferiores à população atual.

Contudo, aponta-se que apesar da fecundidade adolescente relativamente alta, o rejuvenescimento dessas taxas, que vinha ocorrendo há várias décadas, já não ocorre e há tendência de uma pequena postergação. Os diferenciais por educação e rendimento também indicam que a maior redução na fecundidade ocorreu entre mulheres menos educadas e mais pobres, já que entre as com 12 ou mais anos de estudo a TFT é de 1,2 (BERQUÓ e CAVENAGHI, 2014). Com a postergação e aumento da educação entre as mulheres, é provável que a fecundidade continue o caminho de redução, com um cenário futuro possivelmente mais próximo da mediana ou até das tendências mais baixas das projeções.

Ademais, se o tamanho da população será importante, mais ainda será pensar sua distribuição espacial, e dados os processos de urbanização, as tendências de crescimento das cidades, principalmente em termos de tamanho ou forma.

A comparação entre a fecundidade desse início de século e da provável fecundidade do final mostram diferenças claras, já que, com exceção de algumas localidades na África, há uma convergência a valores praticamente abaixo de 2 filhos por mulher 
em 2100 (UN, 2013). Nesse cenário, certamente não será a queda da fecundidade que resolverá os problemas ambientais. Tampouco esse movimento de queda significará necessariamente aumento dos direitos reprodutivos. Nesse sentido as projeções indicam que tanto os problemas ambientais como os relativos à fecundidade precisam ser pensados em suas relações mais amplas, já que nenhum será equacionado se consideradas somente uma de suas dimensões.

\section{Referências}

AGADJANIAN, V.; PRATA, N. War, peace, and fertility in Angola. Demography, v. 39, n. 2, p. 215-231, 2002.

AGGARWAL, R.; NETANYAHU, S.; ROMANO, C. Access to natural resources and the fertility decision of women: The case of South Africa. Environment and Development Economics, v. 6, n. 2, p. 209-236, 2001.

ANDRIETTA, J. Influência da exposição ocupacional à poluição atmosférica de origem veicular nos parâmetros seminais de controladores de tráfego na Região Metropolitana de São Paulo. 2010. Dissertação (Mestrado em Urologia). Faculdade de Medicina. Universidade de São Paulo, 2010.

BERQUÓ, E.; CAVENAGHI, S. M. Tendências dos diferenciais educacionais e econômicos da fecundidade no Brasil entre 2000 e 2010. In: XIX Encontro Nacional de Estudos Populacionais, 2014, São Pedro/SP. Anais... São Pedro: ABEP, 2014.

BIDDLECOM, A. E.; AXINN, W. G.; BARBER, J. S. Environmental effects on family size preferences and subsequent reproductive behavior in Nepal. Population and Environment, v. 26, n. 3, p. 183-206, 2005. 
BILLINGSLEY, S.; FERRARINI, T. Family Policy and Fertility Intentions in 21 European Countries. Journal of Marriage and Family, v. 76, n. 2, p. 428-445, 2014.

BONGAARTS, J. Fertility and reproductive preferences in posttransitional societies. Population and Development Review, v. 27, p. 260-281, 2001. Supplement: Global fertility transition.

BOSERUP, E. Population and Technological Change. Chicago: University of Chicago Press, 1981.

BRANUER-OTTO, S. Environment and Fertility: The Effect of Flora Quality on Fertility Behavior. Population and Environment, v. 36, n. 1, p. 1-31, 2014.

BURDORF, A.; FIGA-TALAMANCA, I.;JENSEN, T.; THULSTRUP, A. Effects of occupational exposure on the reproductive system: core evidence and practical implications. Occupational Medicine, v. 56, n. 8, p. 56:516-520, 2006.

CALDWELL, J. C. Social upheaval and fertility decline. Journal of Family History, v. 29, n. 4, p. 382-406, 2004.

CAVENAGHI, S. Fertility Decline and Public Policies to Address Population Rights: Perspective from Latin America. Population Division, Expert Paper, n. 5, 2013.

COHEN, J. E. How Many People can the Earth Support? Nova York: W.W. Norton and Co, 1995.

CURRAN, S.; DE SHERBININ, A. Completing the Picture: The Challenges of Bringing 'Consumption' into the PopulationEnvironment Equation. Population and Environment, v. 26, n. 2, 2004.

DE SHERBININ, A., CARR, D., CASSELS, S.; JIANG, L. Population and environment. Annual Review of Environment and Resources, v. 32, p. 345-373, 2007.

DUNLOP, A. Green inclined, but missing the target. The Black Sheep Journal, 25 mar. 2013. Disponível em: http://truth-out. 
org/opinion/item/15583-green-inclined-but-missing-thetarget. Acesso em: 15 jan. 2015.

EHRLICH, P. The Population Bomb. Nova York: Random House, 1968.

FILMER, D.; PRITCHETT, L. H. Environmental degradation and the demand for children: Searching for the vicious circle in Pakistan. Economic and Development Economics, v. 7, p. 123146, 2002.

FINLAY, J. E. Fertility response to natural disasters: the case of three high mortality earthquakes. Policy Research Working Paper, n. 4883, The World Bank, 2009.

FOSDICK, B.; RAFTERY, A. Regional probabilistic fertility forecasting by modeling between-country correlations. Demographic Research, v. 30, p. 1011-1034, 2014.

GAUTHIER, A. H. The impact of family policies on fertility in industrialized countries: a review of the literature. Population Research and Policy Review, v.26, n.3, p.323-346, 2007.

GAUTHIER, A. H.; PHILIPOV, D. Can policies enhance fertility in Europe? Vienna yearbook of population research. Vienna, Austria: Austrian Academy of Sciences, 2008.

HAUSER, R.; WILLIAMS, P.; ALTSHUL, L.; CALAFAT, A. M. Evidence of interaction between polychlorinated biphenyls and phthalates in relation to human sperm motility. Environ. Health Perspect, v. 113, n. 5, p. 425-430, 2005.

HILL, K. War, humanitarian crises, population displacement, and fertility: A review of evidence. Washington, DC: National Resource Council, 2004.

HOGAN, D. J. População e meio ambiente: a emergência de um novo campo de estudos. In: HOGAN, D. J. (Org.). Dinâmica populacional e mudança ambiental: cenários para o desenvolvimento brasileiro. Campinas: Nepo/Unfpa, p. 1-25, 2007. 
HOMAN, G.F.; DAVIES, M.; NORMAN, R. The impact of lifestyle factors on reproductive performance in the general population and those undergoing infertility treatment: a review. Hum Reprod Update; v. 13, n. 3, p. 209-223, 2007.

HUMMEL, D.; ADAMO, S.; SHERBININ, A. et al. Inter- and transdisciplinary approaches to population-environment research for sustainability aims: a review and appraisal. Population and Environment, v. 34, n. 4, p. 481-509, 2012.

HUNTER, L. The Environmental Implications of Population Dynamics. RAND, 2001. Disponível em: http://www.rand. org/publications/MR/MR1191/. Acesso em: 10 jan. 2015.

HUNTER, L. The Population and Environment: A complex relationship. RAND, 2000. Disponível em: http://www.rand. org/pubs/research_briefs/RB5045.html. Acesso em: 10 jan. 2015.

HYMAS, L. I decided not having children for environmental reasons. Guardian Environment network. 2011. Disponível em: http://www.theguardian.com/environment /2011/sep/27/ not-have-children-environmental-reasons. Acesso em: 13 jan. 2015.

KOIFMAN, S.; HATAGIMA, A. Agrotóxicos e câncer no Brasil. In: PERES, F.; MOREIRA, J. C. (Eds.). É veneno ou é remédio? 1a ed. Fundação Oswaldo Cruz, Rio de Janeiro, 2003, p. 75-100.

LEVINE, R. J.; BORDSON, B. L.; MATHEW, R. M. Deterioration of semen quality during summer in New Orleans. Fertil Steril, v. 49 , n. 5, p. 900-907, 1988.

LICHTENFELS, A.; GOMES, J.; PIERI, P.; MIRAGLIA, S.; HALLAK, J.; SALDIVA, P. Increased levels of air pollution and a decrease in the human and mouse male-to-female ratio in Sao Paulo, Brazil. Fertility and Sterility, v. 87, n. 1, p. 230-232, 2007. 
LUCI-GREULICH, A.; THÉVENON O. The impact of family policy packages on fertility trends in developed countries. European Journal of Population, v. 29, n. 4, p. 387-419, 2013.

LUTZ, W.; O'NEILL, B.C.; SCHERBOV, S. Demographics. Europe's population at a turning point. Science, v. 299, n. 5915, p. 19911992, 2003.

LUTZ, W.; PRSKAWETZ, A.; SANDERSON, W.C. Introduction. In: LUTZ, W.; PRSKAWETZ, A.; SANDERSON, W.C. (eds.). Population and Environment: methods of analysis. A suplement to Vol. 28, Population and Environment Review, p. 1-21, 2002.

LUTZ, W.; SCHERBOV, S. Exploratory Extension of IIASA's World Population Projections: Scenarios to 2300. IIASA Interim Report IR-08-022. Laxenburg, Austria: IIASA, 2008.

LUTZ, W.; SCHERBOV, S. Quantifying Vicious Circle Dynamics: The PEDA Model for Population, Environment, Development and Agriculture in African Countries. IIASA Interim Report IR-99-049. Laxenburg, Austria: IIASA, 1999.

LUTZ, W.; SKIRBEKK, V. Policies addressing the tempo effect in low-fertility countries. Population and Development Review, v.3 1, n. 4, p. 699-720, 2005.

MARTINE, G. O contraste entre desenvolvimento sustentável na CIPD e a realidade atual. In: WONG, L. R. et al. Cairo +20: perspectivas da agenda de população e desenvolvimento sustentável pós-2014. Rio de Janeiro: ALAP, 2014.

MEADOWS, D. H.; MEADOWS, D. L.; RANDERS, J.; BEHRENS, W. Limits to growth. Universe Books, New York, 1972.

MONTGOMERY, M. R.; COHEN, B. From death to birth: mortality decline and reproductive change. Washington, D.C.: National Academic Press, 1998.

NOBLES, J.; FRANKENBERG, E.; THOMAS, D. The effects of mortality on fertility: population dynamics after a natural disaster. Demography, v. 52, n. 1, p.15-38, January, 2015. 
O'NEILL, B. C.; MACKELLAR, F. L.; LUTZ, W. Population and Climate Change. Cambridge University Press: Cambridge, UK, 2001.

PARADA, B.; REQUIXA, A.; FIGUEIREDO, A.; MOTA, A. Infertilidade Masculina e Factores Ambientais. Acta Urológica, v. 21, n. 4, p. 9-15, 2004.

PARADISI, R.; VANELLA, S.; BARZANTI, R.; CANI, C.; BATTAGLIA, C.; SERACCHIOLI, R.; VENTUROLI, S. Effects of indoor air purification by an air cleaning system (Koala technology) on semen parameters in male factor infertility: results of a pilot study. Andrologia, v. 41, n. 3, p. 163-168, 2009.

PEREZ, S. G. Household demographic factors as life cycle determinants of land use in the Amazon. Population Research and Policy Review, v. 20, n. 3, p. 159-186, 2001.

PHILIPOV, D., O. THÉVENON, J. KLOBAS, L. BERNARDI AND A.C. LIEFBROER. Reproductive decision-making in a macromicro perspective (REPRO): a state of the art review. A working paper of the European Commission within the Seventh Framework Programme under the Socio-economic Sciences and Humanities Theme, 2009.

RAFTERY, A.E.; LI, N.; ŠEVČÍKOVÁ, H.; GERLAND, P.; HEILIG, G. K. Bayesian probabilistic population projections for all countries. Proceedings of the National Academy of Sciences, v. 109, n. 35, p. 13915-13921, 2012.

ROYAL SOCIETY. People and the planet. The Royal Society Science Policy Centre report 01/12, Londres, 2012.

SCHEIKE, T.H.; RYLANDER, L.; CARSTENSEN. L.; KEIDING. N.; JENSEN, T. K.; STROMBERG. U.; JOFFE, M.; AKRE, O. Time trends in human fecundability in Sweden. Epidemiology, v. 19, n. 2, p. 191-196, 2008.

SKAKKEBAEK NE, RAJPERT-DE MEYTS E, MAIN KM. Testicular dysgenesis syndrome: an increasingly common 
developmental disorder with environmental aspects. Human Reproduction, v. 16, n. 5, p. 972-978, 2001.

STRIESSNIG , E.; LUTZ, W. How does education change the relationship between fertility and age-dependency under environmental constraints? A long-term simulation exercise. Demographic Research, v. 30, pp. 465-492, 2014.

SUTHERLAND, E.; CARR, D.; \& CURTIS, S. Fertility and the Environment in a Natural Resource Dependent Economy: Evidence from Petén, Guatemala. Poblacion y Salud en Mesoamérica, v. 2, n. 1, p. 1-14, 2004.

TAS, S.; LAUWERYS, R.; LISON, D. Occupational hazards for the male reproductive system. Critical Review in Toxicology, v. 26, n. 3, p. 261-307, 1996.

UNITED NATIONS (UN). Probabilistic population projections. UN/ DESA, New York, 2013.

UNIVERSIDADE FEDERAL DE JUIZ DE FORA (UFJF). Estilo de vida tem mais peso sobre a fertilidade. 14 jan. 2011. Disponível em: http://www.ufff.br/ladem/2011/01/14/estilo-de-vida-temmais-peso-sobre-a-fertilidade/. Acesso em: 15 jan. 2015.

VANWEY, L. K.; D'ANTONA, A.; BRONDIZIO, E. Household demographic change and land use/land cover change in the Brazilian Amazon. Population and Environment, v. 28, n. 3, p. 163-185, 2007. 